\title{
Dietary chia seed induced changes in hepatic transcription factors and their target lipogenic and oxidative enzyme activities in dyslipidaemic insulin-resistant rats
}

\author{
Andrea S. Rossi†, Maria E. Oliva†, Maria R. Ferreira, Adriana Chicco and Yolanda B. Lombardo* \\ Department of Biochemistry, School of Biochemistry, University of Litoral, Ciudad Universitaria Paraje El Pozo, \\ CC 242, 3000 Santa Fe, Argentina \\ (Submitted 12 December 2011 - Final revision received 6 July 2012 - Accepted 10 July 2012 - First published online 5 September 2012)
}

\section{Abstract}

The present study analyses the effect of dietary chia seed rich in $n-3 \alpha$-linolenic acid on the mechanisms underlying dyslipidaemia and liver steatosis developed in rats fed a sucrose-rich diet (SRD) for either 3 weeks or 5 months. The key hepatic enzyme activities such as fatty acid synthase (FAS), acetyl-CoA carboxylase (ACC), glucose-6-phosphate dehydrogenase (G-6-PDH), carnitine palmitoyltransferase-1 (CPT-1) and fatty acid oxidase (FAO) involved in lipid metabolism and the protein mass levels of sterol regulatory element-binding protein-1 (SREBP-1) and PPAR $\alpha$ were studied. (1) For 3 weeks, Wistar rats were fed either a SRD with $11 \%$ of maize oil (MO) as dietary fat or a SRD in which chia seed replaced MO (SRD + Chia). (2) A second group of rats were fed a SRD for 3 months. Afterwards, half the rats continued with the SRD while for the other half, MO was replaced by chia for 2 months (SRD + Chia). In a control group, maize starch replaced sucrose. Liver TAG and the aforementioned parameters were analysed in all groups. The replacement of MO by chia in the SRD prevented (3 weeks) or improved/normalised ( 5 months) increases in dyslipidaemia, liver TAG, FAS, ACC and G-6-PDH activities, and increased FAO and CPT-1 activities. Protein levels of PPAR $\alpha$ increased, and the increased mature form of SREBP-1 protein levels in the SRD was normalised by chia in both protocols (1 and 2). The present study provides new data regarding some key mechanisms related to the fate of hepatic fatty acid metabolism that seem to be involved in the effect of dietary chia seed in preventing and normalising/ improving dyslipidaemia and liver steatosis in an insulin-resistant rat model.

\section{Key words: Dietary chia seeds: Dyslipidaemia: PPAR $\alpha$ : Sterol regulatory element-binding protein-1}

In the last few years, it has been recognised that the dietary intake of marine PUFA (fish oil rich in 20:5n-3 (EPA) and 22:6n-3 (DHA)) appears to play a key role against the adverse effects of the so-called metabolic syndrome, which includes type 2 diabetes, insulin resistance (IR), dyslipidaemia, hypertension, obesity and $\mathrm{CVD}^{(1-3)}$, among other diseases. Another nutritionally important $n-3$ PUFA is $\alpha$-linolenic acid (ALA, 18:3n-3). In human diets, the essential ALA is usually derived from plant sources. Epidemiological and clinical studies have suggested that a higher consumption of ALA is associated with a reduced risk of $\mathrm{CVD}^{(4-6)}$. Available information indicates that dietary fats rich in ALA such as linseed or perrilla oil, compared with those rich in linoleic acid, decrease serum lipid concentrations in $\operatorname{rats}^{(7-9)}$. One of the richest botanical sources of ALA is the chia seed (Salvia bispanica L. $)^{(10,11)}$. Chia, which is also rich in fibre and minerals, was a major dietary component of the Mayans and Aztecs. Chia seed has also received increasing attention for its potential role in the prevention of dyslipidaemia. In this regard, some animal studies have shown a reduction in plasma TAG, NEFA and total cholesterol and an increase in HDL-cholesterol when this food ingredient was used as a source of dietary fat in normal or dyslipidaemic rats ${ }^{(12,13)}$ On the other hand, normal rats fed a sucrose-rich diet (SRD) developed dyslipidaemia and IR among other biochemical and metabolic alterations that mimic many aforementioned signs of the metabolic syndrome ${ }^{(3,14,15)}$. We have recently demonstrated that feeding these rats for 3 weeks with a SRD in which chia seed was the source of dietary fat prevents the onset of dyslipidaemia and IR without changes in plasma glucose levels. Moreover, dyslipidaemia and IR in rats fed the SRD for a long term $(5$ months) were reversed

\footnotetext{
Abbreviations: ACC, acetyl-CoA carboxylase; ALA, $\alpha$-linolenic acid; CPT-1, carnitine palmitoyltransferase-1; FAO, fatty acid oxidase; FAS, fatty acid synthase; G-6-PDH, glucose-6-phosphate dehydrogenase; IR, insulin resistance; SRD, sucrose-rich diet; SRD + Chia, sucrose-rich diet + chia seed; SREBP-1c, sterol regulatory element-binding protein-1c.
}

* Corresponding author: Professor Dr Y. B. Lombardo, fax +54342 4575221, email ylombard@fbcb.unl.edu.ar

† Both authors contributed equally to the laboratory assays in the present study. 
without changes in plasma insulin levels when chia seed instead of maize oil became the dietary source of fat for the last 2 months of the feeding period ${ }^{(13)}$. In addition, dietary chia seed reduced increased epididymal and retroperitoneal fat pad weights present in long-term SRD-fed rats ${ }^{(13)}$, and induced lipid redistribution and attenuated metabolic cardiovascular and hepatic signs of rats fed a high-carbohydratefat diet ${ }^{(16)}$. Ghafoorunissa et al. ${ }^{(9)}$ showed that in weanling normal rats fed a SRD, the partial substitution of dietary linoleic acid with ALA for 3 months resulted in lowered blood lipid levels and partially corrected sucrose-induced IR.

At present, the mechanisms by which dietary chia seed could normalise or improve altered plasma lipid levels are still not completely understood. It is possible that the proportion of ALA ( $64 \%$ of total lipids) in the chia seed as a precursor of EPA and DHA, similar to the effect of fish oil, could modify fatty acid metabolism in the liver through the activation of PPAR $\alpha$ and the down-regulation of sterol regulatory element-binding protein-1c (SREBP-1c), leading to a decrease in serum lipids.

Therefore, the aim of the present study was to determine whether changes in the fate of hepatic fatty acid metabolism (lipogenesis-oxidation) could play a role in the mechanisms underlying the effect of the dietary intake of chia seed in the prevention/reversion of liver steatosis and dyslipidaemia in rats fed a SRD. To achieve this goal, two experimental protocols were used. Both protocols were designed to investigate the effectiveness of chia seed as a dietary source of fat in SRD-fed rats in either: (1) preventing the development of the aforementioned metabolic abnormalities or (2) improving/ reversing them in the presence of stable dyslipidaemia achieved after long-term SRD feeding.

In both protocols, we measured the activities of the following hepatic key enzymes involved in de novo lipogenesis and fatty acid oxidation: acetyl-CoA carboxylase (ACC); fatty acid synthase (FAS); glucose-6-phosphate dehydrogenase (G-6-PDH); carnitine palmitoyltransferase-1 (CPT-1 - the ratelimiting enzyme for mitochondrial $\beta$-fatty acid oxidation); fatty acid oxidase (FAO - generally used to monitor the activity of PPAR $\alpha$ ). Furthermore, in the liver, we analysed the protein mass levels of the transcription factors SREBP-1, which has a central role in the induction of the gene expression of lipogenic enzymes, and PPAR $\alpha$, which belongs to the superfamily of ligand-activated nuclear hormone receptors and regulates the expression of fatty acid oxidation enzymes.

\section{Materials and methods}

\section{Animals}

Male Wistar rats initially weighing 180-190 g and purchased from the National Institute of Pharmacology were maintained with unrestricted access to water and food under controlled temperature $\left(22 \pm 1^{\circ} \mathrm{C}\right)$, humidity and air flow conditions, with a fixed $12 \mathrm{~h}$ light-dark cycle (lights on from 07.00 to 19.00 hours). They were initially fed a standard non-purified diet (Ralston Purina). After 1 week of acclimatisation, the rats were randomly assigned to one of the two experimental protocols described in the following sections. The experimental protocols were approved by the Human and Animal Research Committee of the School of Biochemistry, University of Litoral, Argentina.

\section{Dietary manipulations}

Experimental protocol 1. A total of twenty-four rats were randomised into three groups and fed an assigned diet for 3 weeks (Table 1). The first group of rats received a semi-synthetic diet containing maize starch (60\% energy), protein (17\% energy) and maize oil as the source of fat (23\% energy) (control diet; CD). The other two groups received the same semi-synthetic diet with sucrose as the carbohydrate and fat provided by maize oil (SRD) or by chia seed (SRD + Chia).

Experimental protocol 2. A total of forty rats were divided into two groups and fed for 3 months with the CD $(n 16)$ or SRD ( $n$ 16) described previously in experimental protocol 1. At that time, rats in the SRD group were randomly subdivided into two subgroups. The first subgroup continued with the SRD up to 5 months of feeding and the second subgroup received the $\mathrm{SRD}+$ Chia as the source of dietary fat for the next 2 months. The control group was fed with the CD throughout the experimental period. The fibre, vitamin mix and salt mix contents of each diet were similar. The carbohydrate, protein and fibre contents in the feed of the SRD + Chia group were balanced taking into account the amount of these nutrients present in the chia seed. The fatty acid composition of the chia seed was provided by the supplier (Table 2). The preparation and handling of the diets have been reported elsewhere ${ }^{(13)}$. The food in the animal cages was shaded from light and changed every day. All diets provided approximately $18.00 \mathrm{~kJ} / \mathrm{g}$ of food. The weight of each animal and the energy intake were recorded twice per week during the experimental period in all groups and subgroups of rats.

At least six rats from the three dietary groups were used in each procedure. They were anaesthetised with intraperitoneal

Table 1. Composition of the experimental diets* (based on the AIN-93 diet)

\begin{tabular}{lccc}
\hline Diet ingredients (\% energy) & CD & SRD & SRD + Chia \\
\hline Carbohydrates & & & \\
$\quad$ Starch & 60 & 2.6 & - \\
$\quad$ Sucrose & - & 57.4 & 57.4 \\
$\quad$ Chia seed & - & - & 2.6 \\
Fat & 23 & 23 & 0.2 \\
$\quad$ Maize oil & - & - & 22.8 \\
$\quad$ Chia seed & 17 & 17 & 9.25 \\
$\quad$ Patein & - & - & 7.75 \\
$\quad$ Chia seed & &
\end{tabular}

CD, control diet; SRD, sucrose-rich diet; SRD + Chia, SRD + chia seed.

${ }^{*}$ All diets contained by weight: salt mix, 3.5\% (AIN-93-MX(25)); vitamin mix, $1 \%$ (AIN-93-VX(25)); choline chloride, $0.2 \%$; methionine, $0.3 \%$; fibre, $12 \%$. The SRD + Chia was balanced in the fibre and salt mix according to the amount of each one in the chia seed provided by the manufacturer.

† Chia seed (Salba; Salvia hispanica L.): $362 \mathrm{~g} / \mathrm{kg}$ diet. Chia composition (g/100 g chia seed): carbohydrate, 37.45 ; insoluble fibre, $81 \%$ of total carbohydrate; fat, 30.23; protein, 21.19. Mineral composition (mg/100 g chia seed): $\mathrm{Na}, 103.15 ; \mathrm{K}$ 826.15; Ca, 589.60; Fe, 11.90; Mg, 77.0; P, 604.0; Zn, 5.32; Cu, 1.66; Mn, 1.36. 
Table 2. Maize oil and chia seed fatty acid composition ( $\mathrm{g} / 100$ total fatty acids)

\begin{tabular}{lcc}
\hline Fatty acids $^{*}$ & Maize oil & Chia seed \\
\hline $16: 0$ & 10.40 & 6.60 \\
$18: 0$ & $2 \cdot 60$ & 2.80 \\
$18: 1 n-9$ & $32 \cdot 10$ & $6 \cdot 80$ \\
$18: 2 n-6$ & 51.50 & 18.60 \\
$18: 3 n-3$ & 0.76 & 64.60 \\
$20: 1 n-9$ & 0.45 & 0.30 \\
Total saturated & 13.40 & 12.04 \\
Monounsaturated & 32.55 & 7.44 \\
Polyunsaturated & & \\
$n-6$ & 51.50 & 18.60 \\
$n-3$ & 0.76 & 64.60 \\
$n-6: n-3$ & 67.80 & 0.287 \\
\hline
\end{tabular}

* Minor components made content up to $100 \%$.

† Salba; Salvia hispanica L. (Agrisalba SA, Buenos Aires, Argentina).

sodium pentobarbital ( $60 \mathrm{mg} / \mathrm{kg}$ body weight). Blood samples were obtained from the jugular vein and rapidly centrifuged; plasma was either immediately assayed or stored at $-20^{\circ} \mathrm{C}$. Liver, epididymal, retroperitoneal and omental adipose tissue were totally removed and weighed. Liver was immediately frozen and stored at the temperature of liquid $\mathrm{N}_{2}$. Visceral adiposity index (\%) was calculated as follows and expressed as adiposity percentage ${ }^{(16)}$ :

$$
\begin{aligned}
& \text { Visceral adiposity index }(\%)=(\text { retroperitoneal fat }(\mathrm{g}) \\
& + \text { omental fat }(\mathrm{g})+\text { epididymal fat }(\mathrm{g})) \text { /body weight }(\mathrm{g}) \\
& \times 100 \text {. }
\end{aligned}
$$

\section{Analytical methods}

Plasma TAG, NEFA and glucose levels were determined by spectrophotometric methods and insulin by immunoreactive assays as described previously ${ }^{(17)}$. The insulin assays were calibrated against rat insulin standard (Novo Nordisk). TAG content was determined in the homogenate of frozen liver, as described previously ${ }^{(18)}$

\section{Enzymatic activity assays}

ACC was assayed according to Zimmermann et al. ${ }^{(19)}$. The cytosolic fraction was obtained after centrifugation of the supernatant at $30000 \mathrm{rpm}$ for $1 \mathrm{~h}$ at $4^{\circ} \mathrm{C}$. ACC was measured by an NADH-linked assay. FAS activity was assayed on the cytosolic fraction of liver tissue by measuring malonyl CoA-dependent oxidation of $\mathrm{NADPH}$ at $37^{\circ} \mathrm{C}$, as described previously ${ }^{(18)}$. Liver tissue G-6-PDH, peroxisomal FAO and CPT-1 were determined as described recently ${ }^{(18)}$.

\section{Western blot analysis of liver protein mass levels of PPAR $\alpha$ and sterol regulatory element-binding protein-1}

Liver homogenates were prepared for PPAR $\alpha$ protein mass level analysis and Western blots were run under reduced-denatured protein (Laemmli buffer) as described by Hein et al. ${ }^{(18)}$. Kidney tissue extracts were employed as PPAR $\alpha$ positive control. Nuclear and microsomal membrane extracts of SREBP-1 from the liver of rats were obtained by centrifugation in a sucrose gradient as described by Ascencio et $a l .{ }^{(20)}$ with slight modifications. Tissue was homogenised in lysis buffer $(0.5 \mathrm{~g}$ tissue $/ 3.75 \mathrm{ml}$ ) containing $0.3 \mathrm{M}$-sucrose, $5 \mathrm{~mm}$-dithiothreitol,

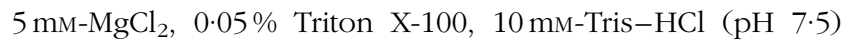
and tissue protease inhibitor cocktail $(5 \mu \mathrm{l} / 100 \mathrm{mg})$. Tissue homogenates were centrifuged at $2000 \mathrm{rpm}$ for $10 \mathrm{~min}$ at $4^{\circ} \mathrm{C}$. The supernatant was used as the microsomal membrane extract. The pellet was resuspended in $2 \mathrm{ml}$ of sucrose buffer containing $2 \cdot 3 \mathrm{~m}$-sucrose, $2 \mathrm{~mm}-\mathrm{MgCl}_{2}, 10 \mathrm{~mm}$-Tris- $\mathrm{HCl}(\mathrm{pH} 7 \cdot 5)$ and tissue protease inhibitor cocktail $(5 \mu \mathrm{l} / 100 \mathrm{mg})$. The suspension was centrifuged at $32000 \mathrm{rpm}$ for $60 \mathrm{~min}$ at $4^{\circ} \mathrm{C}$. The pellet was used as the nuclear extract and resuspended in $600 \mu \mathrm{l}$ of storage buffer containing $50 \%$ glycerol, $2 \mathrm{~mm}-\mathrm{MgCl}_{2}, 0 \cdot 1 \mathrm{~mm}$-EDTA and $50 \mathrm{~mm}$-HEPES $(\mathrm{pH} 7 \cdot 5)$. The extracts were stored at $-70^{\circ} \mathrm{C}$. The protein concentration of the extracts was determined by the Bradford assay. Protein samples were resolved on SDS-PAGE according to Laemmli. Before electrophoresis, all samples were boiled for $2 \mathrm{~min}$ in the presence of $1 \%$ SDS with $200 \mu \mathrm{m}$-dithiothreitol. For immunoblotting, proteins in the SDS-PAGE gel were transferred to polyvinyldifluoride membranes. The membranes were probed with specific antibodies (rabbit anti-PPAR $\alpha$ antibody and rabbit anti-SREBP-1 antibody; Santa Cruz Biotechnology, Inc.), respectively. The blots were then incubated with horseradish peroxidase-linked secondary antibody followed by chemiluminescence detection according to the manufacturer's instructions (Super Signal West Pico chemiluminescence detection; Pierce Biotechnology). $\beta$-Actin was used as a loading control. The intensity of bands was quantified by National Institutes of Health imaging software (http://rsb.info.nih.gov/nih-image/). The relationship between the amount of samples subjected to immunoblotting and the signal intensity observed was linear under the aforementioned conditions. In addition, the liver SREBP-1 analysis showed one electrophoretic band with an approximate molecular weight of $125 \mathrm{kDa}$ and another band of $68 \mathrm{kDa}$; these bands represent the precursor and mature forms of SREBP-1, respectively ${ }^{(21)}$.

\section{Statistical analysis}

Sample sizes were calculated on the basis of measurements previously made with rats fed either a $\mathrm{CD}$ or a $\operatorname{SRD}^{(15,17,18)}$, considering an $80 \%$ power. Results are expressed as means with their standard errors. Statistical comparisons were done transversely between the different dietary groups. Statistical significance between the groups was determined by oneway ANOVA, with one factor (diet) followed by the inspection of all differences between pairs of means by the NewmanKeuls test ${ }^{(22)}$. Differences having $P$ values lower than 0.05 were considered to be statistically significant (SPSS 15.0 for Windows; SPSS, Inc.).

\section{Results}

Body weight gain, energy intake, visceral adiposity index, plasma metabolite levels and liver TAG contents

Body weight and energy intake were carefully monitored in all groups of rats throughout both experimental periods. As shown in Table 3, after 3 weeks on the diet (experimental 
protocol 1), body weight and energy intake did not differ between the groups. The visceral adiposity index was similar in the three dietary groups. The values were as follows (six animals per group): 2.51 (SEM $0 \cdot 12$ ) g in the CD group; $2 \cdot 18$ (SEM 0.10) $\mathrm{g}$ in the SRD group; 2.58 (SEM 0.08) $\mathrm{g}$ in the SRD+Chia group. As previously shown ${ }^{(13)}$, a significant increase in body weight and energy intake occurred in rats fed a SRD from 3 to 5 months compared with those fed a CD (Table 4, experimental protocol 2). However, in spite of a similar energy intake recorded in both the SRD and SRD + Chia groups during the last 2 months of the experimental period (3-5 months), weight gain at 5 months was slightly lower in the latter group. Moreover, the SRD-fed rats showed a significant increase in retroperitoneal, omental and epididymal fat pad weights, and therefore the visceral adiposity index was significantly higher compared with both CD and SRD + Chia groups. Chia seed as a dietary source of fat in the SRD significantly reduced the weight of the aforementioned fat tissues, reaching values similar to those recorded in the CD-fed group. The visceral adiposity index was as follows (six animals per group): 3.73 (SEM 0.10) in the CD group; 6.23 (SEM 0.70) in the SRD group; 4.09 (SEM 0.21) in the SRD + Chia group $(P<0 \cdot 05$, SRD $v$. CD and SRD + Chia $)$.

At the end of 3 weeks on the diet (experimental protocol 1), both plasma TAG and NEFA levels were significantly higher in SRD-fed rats compared with CD-fed rats. The presence of chia seed as the principal dietary source of fat in the SRD group prevented the increase in these parameters. No changes in plasma glucose were recorded in the three dietary groups. Moreover, confirming previous reports ${ }^{(13)}$, hyperinsulinaemia was present in SRD-fed rats. When chia seed replaced maize oil in the SRD, insulin levels were not statistically different from the CD- or SRD-fed animals (Table 3). In agreement with previous publications ${ }^{(13,23)}$, plasma TAG, NEFA and glucose levels were higher in rats fed the SRD for 5 months compared with the age-matched control fed the CD (Table 4). Similar values were observed in rats fed the SRD for 3 months (data not shown). All the variables returned to control values in SRD-fed rats in which chia seed replaced maize oil for the last 2 months of the feeding period (experimental protocol 2). No statistically significant differences in plasma insulin levels were observed at the end of the experimental period between the three dietary groups (Table 4).

Chia seed prevented the enhanced liver TAG content recorded in rats fed the SRD for 3 weeks. Furthermore, the replacement of maize oil by chia seed in the SRD for the last 2 months of the experimental period ( 5 months) significantly lowered the liver TAG content, although the values were still higher than those observed in CD-fed rats (Tables 3 and 4).

\section{Hepatic enzyme activities involved in lipid metabolism}

As shown in Table 5, the liver of SRD-fed rats, compared with that of CD-fed rats, showed an enhancement of ACC, FAS and G-6-PDH activities (key enzymes related to de novo fatty acid synthesis) either after 3 weeks or 5 months on the diet. However, the substitution of maize oil by chia seed as dietary fat in the SRD for 3 weeks was able to prevent these alterations. Furthermore, the activity of G-6-PDH decreased significantly, reaching values below those of the CD group. Besides, a significant reduction in ACC, FAS and G-6-PDH activities (which returned to values similar to those recorded in CDfed rats) was observed in the liver of SRD-fed rats when chia seed replaced maize oil for the last 2 months of the feeding period (Table 5).

On the other hand, Table 6 shows the hepatic activities of key enzymes related to mitochondrial (CPT-1) and peroxisomal (FAO) fatty acid oxidation in the three dietary groups.

Table 3. Body weight, energy intake, plasma metabolite levels and TAG liver of rats fed the control diet (CD), the sucrose-rich diet (SRD) or the SRD + chia seed (SRD + Chia)‡

(Mean values with their standard errors)

\begin{tabular}{|c|c|c|c|c|c|c|}
\hline & \multicolumn{6}{|c|}{ Experimental protocol 1 (3 weeks) } \\
\hline & \multicolumn{2}{|c|}{$C D$} & \multicolumn{2}{|c|}{ SRD } & \multicolumn{2}{|c|}{ SRD + Chia } \\
\hline & Mean & SEM & Mean & SEM & Mean & SEM \\
\hline \multicolumn{7}{|l|}{ Body weight $(\mathrm{g}) \S$} \\
\hline Initial & $189 \cdot 0$ & $2 \cdot 7$ & $190 \cdot 3$ & $3 \cdot 1$ & $185 \cdot 2$ & $3 \cdot 6$ \\
\hline Final & $275 \cdot 3$ & $5 \cdot 3$ & 277.6 & 4.3 & $276 \cdot 0$ & 2.5 \\
\hline \multicolumn{7}{|l|}{ Energy intake (kJ/d) } \\
\hline 0-3 weeks & $290 \cdot 0$ & $18 \cdot 0$ & $312 \cdot 0$ & $23 \cdot 0$ & $309 \cdot 0$ & $17 \cdot 0$ \\
\hline \multicolumn{7}{|l|}{ Plasma\|l } \\
\hline TAG (mM) & 0.68 & 0.05 & $1.52^{\star \star \star}$ & 0.13 & 0.60 & 0.03 \\
\hline NEFA $(\mu \mathrm{M})$ & $252 \cdot 0$ & $17 \cdot 0$ & $595 \cdot 0^{\star \star \star}$ & $24 \cdot 0$ & $245 \cdot 0$ & $14 \cdot 0$ \\
\hline Glucose (mM) & 6.7 & 0.2 & 6.8 & 0.3 & $7 \cdot 3$ & 0.5 \\
\hline Insulin (pM) & $416 \cdot 7$ & $25 \cdot 0$ & $584.8 \dagger$ & $42 \cdot 6$ & 548.6 & $61 \cdot 8$ \\
\hline \multicolumn{7}{|l|}{ Liver\| } \\
\hline TAG ( $\mu \mathrm{mol} / \mathrm{g}$ wet weight) & 9.9 & 0.5 & $18 \cdot 1^{\star \star \star}$ & $1 \cdot 2$ & $10 \cdot 1$ & 0.6 \\
\hline
\end{tabular}

${ }^{\star \star \star}$ Mean values were significantly different from those of the CD and SRD + Chia groups $(P<0.001)$.

† Mean value was significantly different from that of the CD group $(P<0.05)$.

$\ddagger$ For details of procedures and diets, see the Materials and methods section and Table 1.

$\S n 24$.

|| $n 6$. 
Table 4. Body weight, energy intake, plasma metabolite levels and TAG liver of rats fed the control diet (CD), the sucrose-rich diet (SRD) or the SRD + chia seed (SRD + Chia) $\ddagger$

(Mean values with their standard errors)

\begin{tabular}{|c|c|c|c|c|c|c|}
\hline & \multicolumn{6}{|c|}{ Experimental protocol 2 (5 months) } \\
\hline & \multicolumn{2}{|c|}{ CD } & \multicolumn{2}{|c|}{ SRD } & \multicolumn{2}{|c|}{ SRD + Chia } \\
\hline & Mean & SEM & Mean & SEM & Mean & SEM \\
\hline \multicolumn{7}{|l|}{ Body weight $(\mathrm{g}) \S$} \\
\hline Initial & 184.3 & 2.9 & $185 \cdot 3$ & 4.4 & 183.4 & $3 \cdot 2$ \\
\hline 3 months & 362.4 & $5 \cdot 1$ & 375.5 & $4 \cdot 2$ & 371.3 & $5 \cdot 8$ \\
\hline Final & $430 \cdot 0$ & $7 \cdot 1$ & $490 \cdot 2 \dagger \dagger \dagger$ & $8 \cdot 1$ & $475 \cdot 1+\dagger$ & $10 \cdot 0$ \\
\hline \multicolumn{7}{|l|}{ Energy intake (kJ/d) } \\
\hline $0-3$ months & 288.4 & 12.5 & $295 \cdot 3$ & $9 \cdot 3$ & $291 \cdot 2$ & $10 \cdot 5$ \\
\hline 3-5 months & 289.0 & $10 \cdot 0$ & $355 \cdot 0+\dagger \dagger$ & $6 \cdot 0$ & $348.0+\dagger$ & $12 \cdot 0$ \\
\hline \multicolumn{7}{|l|}{ Plasma\|l } \\
\hline TAG (mM) & 0.70 & 0.07 & $1 \cdot 85^{\star \star \star}$ & 0.12 & 0.65 & 0.07 \\
\hline NEFA $(\mu \mathrm{M})$ & $305 \cdot 0$ & $13 \cdot 0$ & $735 \cdot 0^{\star \star \star}$ & $36 \cdot 0$ & $339 \cdot 0$ & $18 \cdot 0$ \\
\hline Glucose (mм) & 6.9 & 0.1 & $8 \cdot 3^{\star \star \star}$ & 0.2 & $7 \cdot 2$ & 0.2 \\
\hline Insulin (pM) & $504 \cdot 2$ & $33 \cdot 3$ & $526 \cdot 4 \dagger$ & $43 \cdot 7$ & $625 \cdot 0$ & $63 \cdot 2$ \\
\hline \multicolumn{7}{|l|}{ Liver\| } \\
\hline TAG ( $\mu \mathrm{mol} / \mathrm{g}$ wet weight) & $12 \cdot 1$ & 0.9 & $25 \cdot 2^{\star \star \star}$ & 1.3 & $16 \cdot 8+\dagger$ & $1 \cdot 1$ \\
\hline
\end{tabular}

Mean values were significantly different from those of the CD and SRD + Chia groups: ${ }^{\star \star \star} P<0.001$.

Mean values were significantly different from those of the CD group: $+\dagger P<0.01$, + t† $P<0.001$.

$\ddagger$ For details of procedures and diets, see the Materials and methods section and Table 1.

$\S n 40$.

$\| n 6$.

Chia seed as a source of dietary fat was able to prevent the decreased activity of CPT- 1 observed in rats fed the SRD for 3 weeks, while FAO activity significantly increased $(P<0.05)$, reaching values above those recorded in the $\mathrm{CD}$ and SRD groups. Similarly, the presence of chia seed as dietary fat reverted the decreased CPT-1 activity recorded in rats fed the SRD for 5 months, reaching values above those observed in age-matched controls fed the CD. Moreover, FAO activity was also significantly increased in the SRD + Chia group. Interestingly, compared with the CD-fed group, no changes in FAO activity were observed in rats fed the SRD either for a short term (3 weeks) or 5 months in which maize oil was the source of dietary fat (Table 6).
Liver sterol regulatory element-binding protein-1 protein mass levels in the membrane fraction (precursor form) and nuclear extracts (mature form)

To examine whether or not chia seed feeding could alter the SREBP-1 protein mass, the precursor and mature forms of SREBP-1 protein mass levels in the liver of SRD- and SRD + Chia-fed rats were measured by immunoblotting. Since the antibody of SREBP-1 reacted with both SREBP-1a and SREBP-1c forms, we could not distinguish these two forms and thus used the non-committal term SREBP-1 (in the mouse liver, the ratio of SREBP-1c:1a transcriptors was $\left.9: 1^{(21)}\right)$. The 125 and $68 \mathrm{kDa}$ proteins observed represent the

Table 5. Acetyl-CoA carboxylase (ACC), fatty acid synthase (FAS) and glucose-6-phosphate dehydrogenase $(\mathrm{G}-6-\mathrm{PDH})$ activities in the liver of rats fed the control diet (CD), the sucrose-rich diet (SRD) or the SRD + chia seed (SRD + Chia) $\ddagger$

(Mean values with their standard errors; $n 6$ per group)

\begin{tabular}{|c|c|c|c|c|c|c|c|}
\hline \multirow[b]{2}{*}{ Diets } & \multirow[b]{2}{*}{ Time of diet } & \multicolumn{2}{|c|}{$\begin{array}{c}\mathrm{ACC} \text { (pkatal/mg } \\
\text { protein) }\end{array}$} & \multicolumn{2}{|c|}{$\begin{array}{l}\text { FAS (pkatal/mg } \\
\text { protein) }\end{array}$} & \multicolumn{2}{|c|}{$\begin{array}{c}\text { G-6-PDH } \\
\text { (pkatal/mg } \\
\text { protein) }\end{array}$} \\
\hline & & Mean & SEM & Mean & SEM & Mean & SEM \\
\hline \multicolumn{8}{|c|}{ Experimental protocol 1} \\
\hline $\mathrm{CD}$ & 3 weeks & $591 \cdot 2$ & 28.4 & $182 \cdot 0$ & $20 \cdot 1$ & $389 \cdot 1$ & $8 \cdot 3$ \\
\hline SRD & 3 weeks & $818 \cdot 3^{*}$ & $41 \cdot 7$ & $305 \cdot 6^{\star \star \star}$ & $11 \cdot 7$ & $477 \cdot 6^{\star \star}$ & $18 \cdot 4$ \\
\hline SRD + Chia & 3 weeks & $656 \cdot 3$ & $40 \cdot 1$ & $217 \cdot 1$ & $5 \cdot 0$ & $235.5+\dagger$ & $6 \cdot 7$ \\
\hline \multicolumn{8}{|c|}{ Experimental protocol 2} \\
\hline $\mathrm{CD}$ & 5 months & 731.5 & 41.7 & $118 \cdot 6$ & 3.3 & $329 \cdot 0$ & 33.4 \\
\hline SRD & 5 months & $1543 \cdot 1^{* * *}$ & $36 \cdot 8$ & $217 \cdot 1^{\star \star *}$ & $6 \cdot 7$ & $465 \cdot 9^{\star \star \star}$ & $31 \cdot 7$ \\
\hline SRD + Chia & 5 months & $699 \cdot 7$ & $123 \cdot 6$ & 131.9 & $5 \cdot 0$ & $309 \cdot 0$ & $20 \cdot 0$ \\
\hline
\end{tabular}

Mean values were significantly different from those of the CD and SRD + Chia groups: ${ }^{\star} P<0.05,{ }^{\star \star} P<0.01,{ }^{\star \star \star} P<0.001$.

Mean value was significantly different from that of the CD group: $t+P<0.01$.

$\ddagger$ For details of procedures and diets, see the Materials and methods section and Table 1. 
Table 6. Carnitine palmitoyltransferase-1 (CPT-1) and fatty acid oxidase (FAO) activities in the liver of rats fed the control diet (CD), the sucrose-rich diet (SRD) or the SRD + chia seed (SRD + Chia)§

(Mean values with their standard errors; $n 6$ per group)

\begin{tabular}{|c|c|c|c|c|c|}
\hline \multirow[b]{2}{*}{ Diets } & \multirow[b]{2}{*}{ Time of diet } & \multicolumn{2}{|c|}{$\begin{array}{c}\mathrm{CPT}-1 \\
\text { (pkatal/mg } \\
\text { protein) }\end{array}$} & \multicolumn{2}{|c|}{$\begin{array}{c}\mathrm{FAO} \\
\text { (pkatal/mg } \\
\text { protein) }\end{array}$} \\
\hline & & Mean & SEM & Mean & SEM \\
\hline \multicolumn{6}{|c|}{ Experimental protocol 1} \\
\hline CD & 3 weeks & $32 \cdot 9$ & $3 \cdot 3$ & $48 \cdot 0$ & 4.5 \\
\hline SRD & 3 weeks & $17 \cdot 2^{*}$ & $2 \cdot 2$ & 44.8 & $4 \cdot 6$ \\
\hline SRD + Chia & 3 weeks & 31.6 & $4 \cdot 1$ & $68.5 \ddagger$ & $7 \cdot 6$ \\
\hline \multicolumn{6}{|c|}{ Experimental protocol 2} \\
\hline CD & 5 months & $18 \cdot 9$ & $2 \cdot 2$ & $28 \cdot 0$ & 1.7 \\
\hline SRD & 5 months & $11 \cdot 7^{\star \star}$ & $1 \cdot 7$ & $27 \cdot 3$ & $2 \cdot 3$ \\
\hline SRD + Chia & 5 months & $28.4 \dagger \dagger$ & $1 \cdot 8$ & $51 \cdot 8 \ddagger$ & $4 \cdot 8$ \\
\hline
\end{tabular}

Mean values were significantly different from those of the CD and SRD + Chia groups: ${ }^{\star} P<0.05,{ }^{\star \star} P<0.01$.

Mean value was significantly different from that of the CD group: $\dagger \uparrow P<0.01$.

Mean values were significantly different from those of the $C D$ and SRD groups: $\ddagger P<0.05$.

$\S$ For details of procedures and diets, see the Materials and methods section and Table 1.

precursor and mature forms of SREBP-1. Each gel contained an equal number of samples from the CD, SRD and SRD + Chia groups (Fig. 1(a) and (b)). After densitometry of immunoblots, the precursor and mature forms of SREBP-1 of the CD group were normalised to $100 \%$ and both SRD and SRD + Chia were expressed relative to this. The quantitative and qualitative analysis of Western blots showed no changes in the relative abundance of the precursor form of SREBP-1 in the three dietary groups either after 3 weeks or 5 months on the SRD compared with CD-fed rats (Fig. 1(a), experimental protocols 1 and 2). However, the relative abundance of the mature form of the SREBP-1 protein was significantly increased $(P<0.05)$ in SRD-fed rats either after 3 weeks or 5 months on the diet (Fig. 1(b), experimental protocols 1 and 2). The substitution of maize oil by chia seed in the SRD for 3 weeks prevented the increase in the mature form of SREBP-1 protein mass levels (values were similar to those of the CD group), and when chia seed replaced maize oil for the last 2 months in rats fed the SRD for 5 months, the relative abundance of the mature form of SREBP-1 protein mass levels returned to the control values (Fig. 1(b), experimental protocols 1 and 2). The ratios of mature:precursor relative protein levels of SREBP-1 (six animals per group) after the 3-week feeding period were 1.35 (SEM $0 \cdot 11$ ) in the SRD group $v .0 .94$ (SEM 0.05) in the SRD+Chia group $(P<0.01)$, and after 5 months on the diet 1.21 (SEM 0.04) in the SRD group $v .0 .93$ (SEM 0.08) in the SRD+Chia group $(P<0 \cdot 01)$.

\section{Protein mass levels of PPAR $\alpha$}

Since the activities of CPT-1 and FAO were modified in the liver of both SRD and SRD + Chia groups either after 3 weeks or 5 months on the diet, we examined the protein mass levels of the nuclear receptor PPAR $\alpha$. The immunoblotting of liver tissue revealed a single $55 \mathrm{kDa}$ band consistent with PPAR $\alpha$. Each gel contained an equal number of samples from the CD, SRD and SRD + Chia groups (Fig. 2). After densitometry of immunoblots, PPAR $\alpha$ of the CD group was normalised to $100 \%$ and both SRD and SRD + Chia were expressed relative to this. The quantitative and qualitative abundance of Western blot showed that the relative abundance of PPAR $\alpha$ protein levels significantly decreased $(P<0.05)$ in the liver of the SRD group either after 3 weeks or 5 months on the diet compared with rats fed the CD (Fig. 2). The replacement of maize oil by chia seed as the dietary source of fat was able to prevent the decrease in PPAR $\alpha$ protein levels (3 weeks) in SRD-fed rats or even significantly increased $(P<0.05)$ in rats fed the SRD for 5 months (Fig. 2), reaching values above those of the $\mathrm{CD}$ group $(P<0 \cdot 05, \mathrm{SRD}+$ Chia $v$. CD).

\section{Discussion}

Extending our previous research, the present study provides new information concerning some of the possible underlying mechanisms sustaining the beneficial effects of chia seed as a dietary source of fat in the prevention, improvement and/or reversal of dyslipidaemia and liver steatosis that developed in rats rendering dyslipidaemic and insulin resistant by feeding them with a SRD.

The major new findings arising from these investigations are as follows. (1) Dietary chia seed simultaneously administered with a SRD for 3 weeks (experimental protocol 1) prevented the onset of dyslipidaemia and the increase in liver TAG storage (liver steatosis) present in SRD-fed rats by significantly decreasing both the protein mass levels of the mature form of SREBP-1 (without changes in the protein mass level of the precursor form) and the activities of key enzymes (ACC, FAS and G-6-PDH) involved in hepatic de novo lipogenesis, reaching values that did not differ from those observed in the control group fed a CD. In addition, dietary chia seed was able to normalise the low protein mass levels of PPAR $\alpha$ and the activity of the enzyme (CPT-1) involved in mitochondrial fatty acid oxidation; at the same time, it significantly increased the FAO activity involved in peroxisomal fatty acid oxidation, reaching values above those recorded in the CD-fed group. (2) The significant increase in both the protein mass levels of the mature form of SREBP-1 and the enzymatic activities of hepatic ACC, FAS and G-6-PDH observed in rats fed a SRD for 5 months were completely normalised by shifting the source of fat in the SRD from maize oil to chia seed for the last 2 months of the experimental period (experimental protocol 2). Under these experimental conditions, chia seed increased both the low CPT-1 and FAO activities by 1.5- and 2-fold above the value observed in the CD-fed group, respectively. Moreover, dietary chia seed increased the protein mass levels of PPAR $\alpha$, reaching values significantly higher than those recorded in the $\mathrm{CD}$ group. This was accompanied by a significant reduction in hepatic TAG content, the visceral adiposity index without significant changes in energy intake and total body weight and, as previously shown, a normalisation of dyslipidaemia and plasma glucose levels despite the fact that there were no changes in insulinaemia. 
(a)
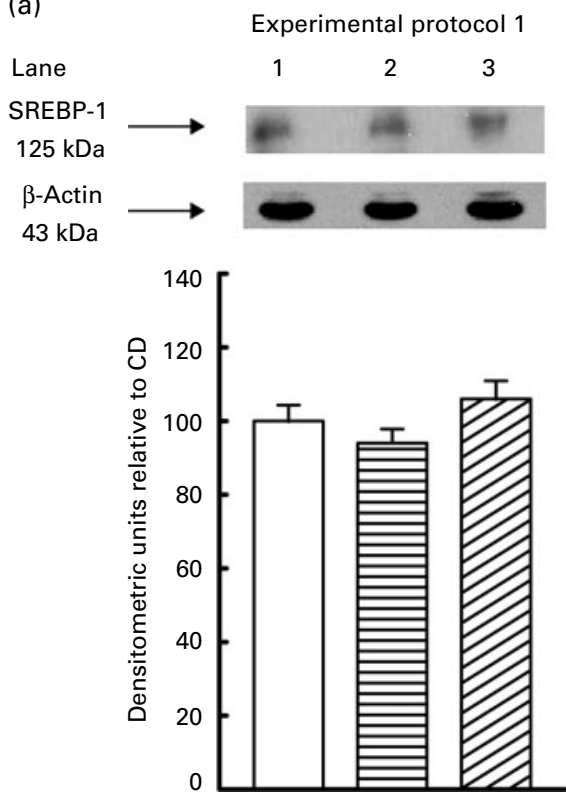

(b)
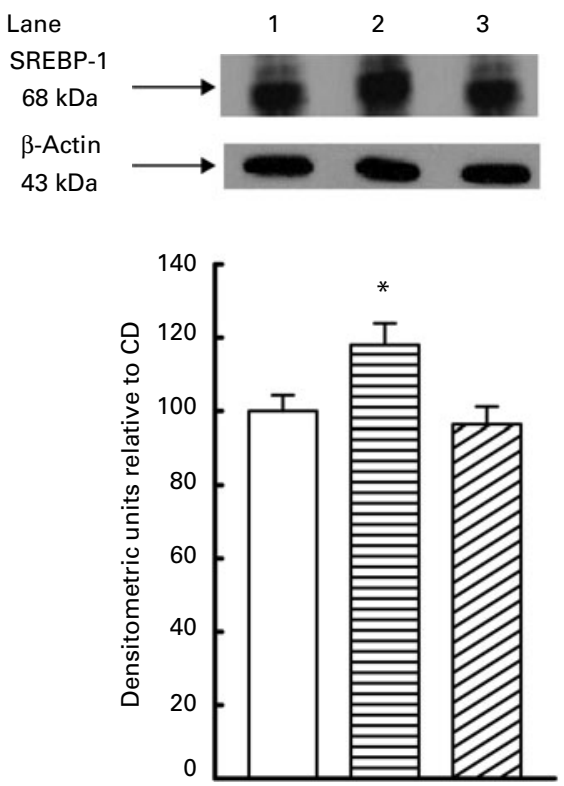

Experimental protocol 2
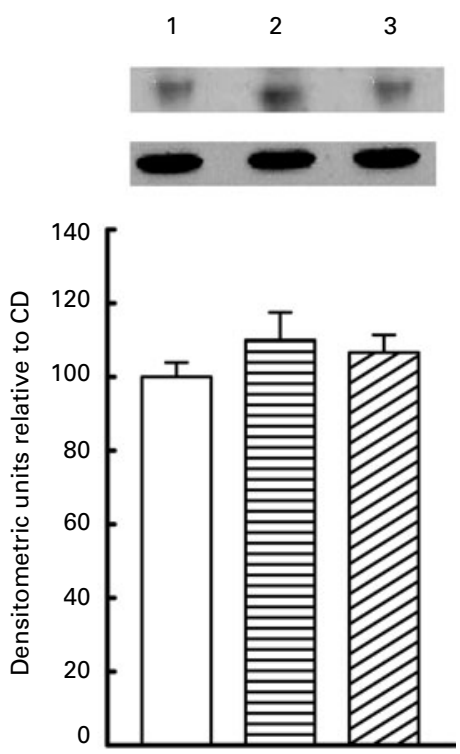

Experimental protocol 2

$\begin{array}{lll}1 & 2 & 3\end{array}$
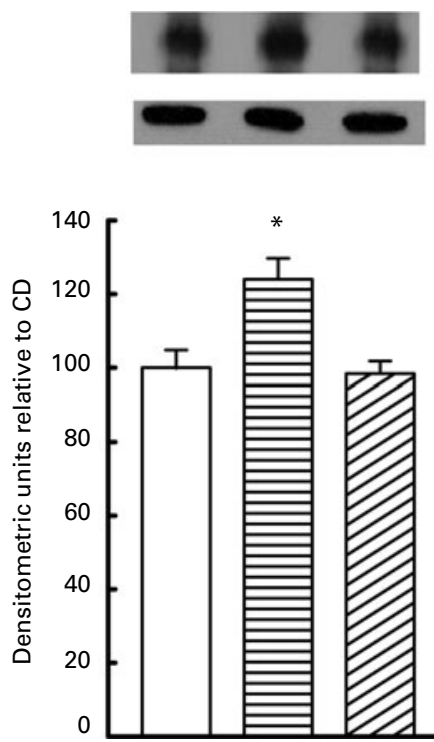

Fig. 1. Liver protein mass levels of (a) the precursor and (b) mature forms of sterol regulatory element-binding protein-1 (SREBP-1) of rats fed a control diet $(C D, \square)$, a sucrose-rich diet (SRD, 目) or a SRD + chia seed (SRD + Chia, $\square$ ). (a) Experimental protocols 1 and 2. Top: a representative immunoblot of the liver precursor form of SREBP-1 from the CD-, SRD- and SRD + Chia-fed rats. Molecular marker is shown on the right. Lane 1, CD; lane 2, SRD; lane 3, SRD + Chia. Bottom: Densitometric immunoblot analysis of the precursor form of SREBP-1 protein mass levels in liver tissue of rats fed a CD, SRD or SRD + Chia. (b) Experimental protocols 1 and 2. Top: a representative immunoblot of the liver mature form of SREBP-1 from the CD-, SRD- and SRD + Chia-fed rats. Molecular marker is shown on the right. Lane 1, CD; lane 2, SRD; lane 3, SRD + Chia. Bottom: Densitometric immunoblot analysis of the mature form of SREBP-1 protein mass levels in the liver tissue of rats fed a CD, SRD or SRD + Chia. Values are means (six animals per group), with their standard errors represented by vertical bars, and expressed as a percentage relative to each $\mathrm{CD}$, respectively. ${ }^{*}$ Mean values were significantly different from those of the $\mathrm{CD}$ and SRD + Chia groups $(P<0.05)$.

Different studies including ours have demonstrated that in rats fed a high-sucrose or high-fructose diet for a short (3-5 weeks) or long (4-8 months) period, a combination of increased VLDL-TAG secretion and a defective removal mechanism of TAG from the circulation lead to a substantial hypertriacylglycerolaemia. This was accompanied by a significant increase in TAG storage within the liver tissue $^{(3,24)}$. The main transcription factor controlling lipid synthesis in the liver is SREBP-1. Several pieces of evidence suggested that the SREBP-1c isoform has a central role in the induction of the gene expression of a number of lipogenic enzymes including ACC and FAS, among others ${ }^{(25,26)}$. In this regard, hepatic gene expression of SREBP-1, protein mass levels and the gene expression and activities of lipogenic 

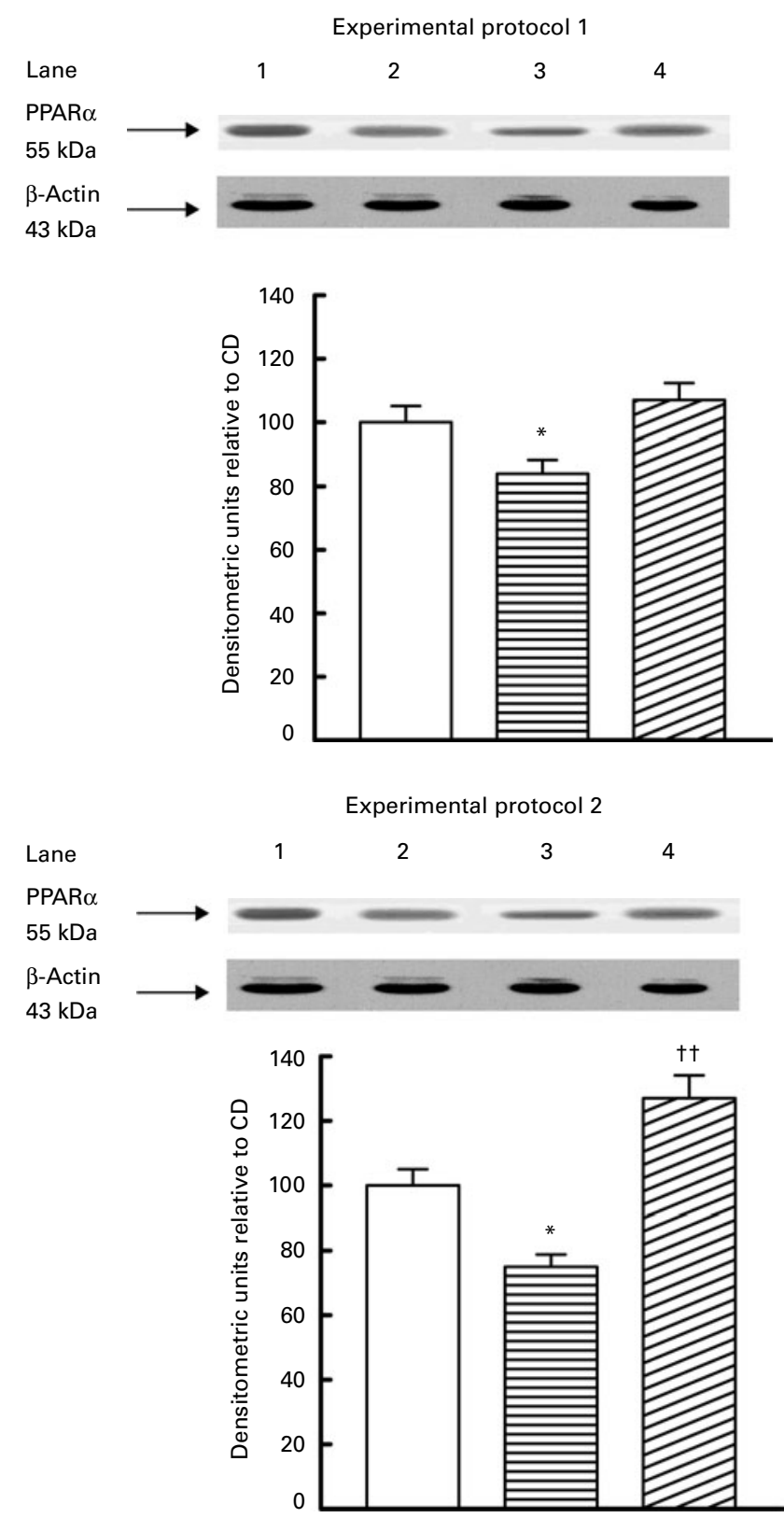

Fig. 2. Liver protein mass levels of PPAR $\alpha$ of rats fed a control diet (CD, $\square$ ), a sucrose-rich diet (SRD, 目) or a SRD + chia seed (SRD + Chia, $\mathbb{Z}$ ). Experimental protocols 1 and 2. Top: a representative immunoblot of liver PPAR $\alpha$ from the CD-, SRD- and SRD + Chia-fed rats. Molecular marker is shown on the right. Lane 1, kidney tissue as a positive control; lane 2, CD; lane 3, SRD; lane 4, SRD + Chia. Bottom. Densitometric immunoblot analysis of PPAR $\alpha$ protein mass levels in the liver tissue of rats fed a CD, SRD or SRD + Chia. Values are means (six animals per group), with their standard errors represented by vertical bars, and expressed as a percentage relative to the $C D$. * Mean values were significantly different from those of the $C D$ and SRD + Chia groups $(P<0 \cdot 05)$. †† Mean values were significantly different from those of the CD group $(P<0.01)$.

enzymes were increased in a high-sucrose/fructose $\operatorname{diet}^{(18,27)}$. In contrast, the mRNA contents and protein mass levels of PPAR $\alpha$ as well as the gene expression and activities of key enzymes of fatty acid oxidation were significantly decreased in rats given a sucrose/fructose-rich diet for different lengths of time (2-8 months) $)^{(18,27)}$.
The present results are in agreement with the aforementioned studies showing that both hepatic lipogenic and oxidative mitochondrial fatty acid oxidation enzyme activities are coordinately increased and decreased, and this was accompanied by a parallel increase and decrease in the protein mass levels of SREBP-1 and PPAR $\alpha$, respectively. This suggests that sucrose induces a shift in the metabolic fate of lipid - synthesis $v$. oxidation - which could be one of the mechanisms involved in increased TAG storage within the liver cells leading to hepatic steatosis in rats fed a SRD. Interestingly, all these changes were expressed not only after longterm SRD feeding but early after 3 weeks on the diet.

On the other hand, it has been shown that $n$-3 PUFA (e.g. EPA, DHA) in vivo or in cell culture inhibited the transcription of gene encoding for key lipogenic enzymes such as ACC, FAS, SCD-1, among others ${ }^{(1,25,28,29)}$. Increases in cellular $n-3$ EPA have also resulted from ingestion of vegetable sources rich in ALA such as flaxseed and perrilla oils and chia seed $^{(8,13,30)}$. ALA could be converted to long-chain $n-3$ PUFA (mainly EPA) and to a lesser extent to DHA in the liver. It is well known that both the absolute amount of ALA and the ratio LA:ALA influence the ALA conversion to EPA as a result of the known competition between $n-6$ and $n$ - 3 fatty acids for desaturation since LA reduces $\Delta-6$ desaturase ${ }^{(31)}$. The ratio of LA:ALA in the SRD + Chia group was $0.287 v .67 \cdot 8$ in the SRD group (maize oil as dietary fat), and Jeffery et $a{ }^{(32)}$ recorded that the maximum hypertriacylglycerolaemic and hypercholesterolaemic effects were obtained at a LA:ALA ratio of 0.33 . To the best of our knowledge, the present study is the first one to demonstrate that in dyslipidaemic, insulin-resistant SRD-fed rats, the higher amount of ALA in the dietary chia seed, or their metabolites EPA and, to a minor extent, DHA, opposite to LA, prevents the increase in both the hepatic lipogenic enzyme activities of ACC, FAS and G-6-PDH and the mature form of SREBP-1 protein mass levels. Moreover, when chia seed replaced maize oil for the last 2 months of the 5-month SRD feeding period, the normalisation of hepatic ACC, FAS and G-6-PDH activities was accompanied by the decrease in the mature form of SREBP-1 protein mass levels. Interestingly, either under the 3-week or 5-month feeding period, the precursor form of SREBP-1 protein mass levels remained unchanged. Therefore, the ratio of mature:precursor relative protein levels that increased in SRD-fed rats returned to normal in the SRD + Chia group. In addition, the concomitant administration of dietary chia seed for 3 weeks enhanced the activity of FAO, contributing to the prevention of the increases in hepatic TAG content by increased peroxisomal fatty acid oxidation. It also prevents the decreased activity of CPT- 1 observed in SRD-fed rats, without significant changes in the protein mass levels of PPAR $\alpha$. The latter was an unexpected finding since FAO enzymatic activity is generally used to monitor the activity of PPAR $\alpha^{(18)}$. However, Neschen et al. ${ }^{(33)}$ showed a significant increase in hepatic peroxisome and both FAO mRNA and 3 ketoacyl-CoA mRNA without changes in the expression of PPAR $\alpha$ and CPT- 1 in rats fed for 3 weeks a high-fat diet containing 59\% of fat-derived energy as fish oil. On the other hand, increased PPAR $\alpha$ protein levels and 
higher activities of the key enzymes of mitochondrial (CPT-1) and peroxisomal (FAO) fatty acid oxidation were recorded when chia seed replaced maize oil for the last 2 months of the 5-month SRD feeding period. These results clearly suggest that the opposite hepatic changes induced by dietary chia seed in the activities of key enzymes involved in lipogenesis and fatty acid oxidation, accompanied by similar changes in the protein mass levels of mature SREBP-1 and PPAR $\alpha$, could be involved in the mechanisms leading to a reduction in liver TAG synthesis, normalising/improving liver steatosis and dyslipidaemia (TAG and NEFA reached control values) in rats fed a SRD. Interestingly, Poudyal et al. ${ }^{(16)}$ recently demonstrated a decrease in hepatic steatosis and reduced fibrosis and inflammation in the histological analysis of the liver of rats fed a high-carbohydrate-high-fat diet supplemented with $5 \%$ of chia seed for 8 weeks. Since ALA is metabolised to EPA and DHA, it is also possible that these metabolites rather than ALA per se could induce the aforementioned changes. In this regard, in mice fed a high-carbohydrate-fat-free diet, Yahagi et al. ${ }^{(25)}$ showed that EPA ethyl ester-specific suppression of liver lipogenic enzymes is mediated by the reduction of mature SREBP-1c protein. Moreover, in B57BL/6J mice, Nakatani et $a l{ }^{(21)}$ showed that low fish oil - rich in EPA and DHA - feeding inhibits the SREBP-1 proteolytic cascade, while a high increase in fish oil decreases mRNA SREBP-1 levels in the liver. $\mathrm{Kim}^{(30)}$ showed a reduction in the activities of FAS, G-6-PDH and malic enzyme and mRNA FAS expression in the liver of rats fed perrilla oil compared with those fed beef tallow, while Ide et al. ${ }^{(34,35)}$ showed that normal rats fed $15 \%$ of perrilla oil for $15 \mathrm{~d}$ significantly increased the mRNA levels and activities of CPT-1 and FAO compared with those of age-matched controls fed safflower oil.

In addition to the prevention and/or normalisation of dyslipidaemia and the increase in liver TAG content, dietary chia seed was able to normalise plasma glucose levels without changes in insulinaemia in rats fed the SRD for a long time (5 months). Moreover, the whole-body peripheral IR present in rats fed a SRD was prevented (3 weeks) or completely normalised ( 5 months) under chia seed administration ${ }^{(13)}$. This effect could also be related to the subsequent changes in fatty acid content in membrane phospholipids of insulin target tissues including liver tissue, due to the absolute and relative amounts of LA and ALA in the diet and the competitive interactions in the metabolism of LA and ALA to long-chain $n-6$ and $n-3$ PUFA, which could in turn improve insulin sensitivity. Interestingly, a high content of EPA and DHA was observed in the hepatic membrane of rats fed perrilla oil, although this oil contained neither EPA nor DHA ${ }^{(7)}$. In this regard, we have recently demonstrated a significant increase in plasma ALA, EPA and DHA in rats fed a SRD when chia seed replaced maize oil as the source of dietary fat ${ }^{(13)}$.

On the other hand, the present results extend our previous observations and show that the increased visceral adiposity index recorded in long-term SRD-fed rats was significantly reduced in the SRD + Chia group, and this effect is not related to energy differences. Okuno et al. ${ }^{(36)}$ also observed a reduction of fat pad in rats fed perrilla oil compared with those fed a saturate fat or LA-rich fat diet, and Poudyal et $a l .{ }^{(16)}$ showed a reduction in the visceral adiposity index due to a decrease in both retroperitoneal and omental but not epididymal fat pad in rats fed a high-carbohydrate-highfat diet supplemented with $5 \%$ of chia seed. Moreover, in rats fed diets containing $0.095-6.3 \%$ of ALA and constant n-6 PUFA levels for 3 weeks, Muhlhausler et al. ${ }^{(37)}$ have recently shown that fat depots in different anatomical locations have different degrees of responsiveness to altered levels of $n$ - 3 and $n$ - 6 long-chain PUFA in the body.

In brief, the present study provides new data regarding some key mechanisms related to the fate of hepatic fatty acid metabolism (lipogenesis/oxidation) that could be involved in the effect of dietary chia seed in preventing, normalising or improving dyslipidaemia, liver steatosis and altered glucose homeostasis in a dyslipidaemic insulin-resistant rat model. Finally, these results, although promising, warrant further research before extrapolating the use of chia seed as a complementary nutrient that could help in the treatment of some signs of the metabolic syndrome in humans, especially considering the few human studies published so far. Interestingly, in healthy individuals receiving $0,7 \cdot 5,15$ or $25 \mathrm{~g}$ of Salba (S. hispanica L.), baked into white bread, Vuksan et al. ${ }^{(38)}$ have recently shown a reduction in postprandial glucose excursion and a prolongation of satiety independently of the amount of Salba used. In addition, they have previously shown a reduction in systolic blood pressure, coagulation and inflammatory markers after 12 weeks of dietary Salba supplement in well-controlled subjects with type 2 diabetes ${ }^{(39)}$. Muramatsu et al. ${ }^{(40)}$ showed that a higher ALA intake was significantly associated with a lower prevalence of IR in middle-aged, normal-weight Japanese individuals, both male and female.

\section{Acknowledgements}

A preliminary report was presented at the 29th International Symposium on Diabetes and Nutrition of the Nutrition Study Group of EASD, June 2011, Rome, Italy. The present study was carried out with the financial support of CONICET and University of Litoral (grants PIP 0105/2010 and CAI + D PI-8-37/2009). The authors thank Agrisalba SA, Buenos Aires, Argentina for providing the chia seed Salba and Mr Walter Da Ru for his skillful technical assistance. A. S. R. was involved in the analysis of hepatic enzyme activities and protein mass levels of PPAR $\alpha$; M. E. O. was involved in the analysis of the precursor and mature forms of SREBP-1 and contributed to the analysis of the results. M. R. F. was involved in the determination of the visceral adiposity index and analytical methods. A. C. was involved in the discussion of the results and the whole manuscript. Y. B. L. wrote the manuscript and discussed it with the whole group of authors. The authors declare that there is no conflict of interest.

\section{References}

1. Clarke SD (2001) Polyunsaturated fatty acid regulation of gene transcription: a molecular mechanism to improve the metabolic syndrome. J Nutr 131, 1129-1132. 
2. Connor WE (2000) Importance of $n$-3 fatty acids in health and disease. Am J Clin Nutr 71, 171S-175S.

3. Lombardo YB \& Chicco AG (2006) Effects of dietary polyunsaturated $n-3$ fatty acids on dyslipidemia and insulin resistance in rodents and humans. A review. J Nutr Biochem 17, $1-13$.

4. de LM, Renaud S, Mamelle N, et al. (1994) Mediterranean alpha-linolenic acid-rich diet in secondary prevention of coronary heart disease. Lancet 343, 1454-1459.

5. Mozaffarian D, Ascherio A, Hu FB, et al. (2005) Interplay between different polyunsaturated fatty acids and risk of coronary heart disease in men. Circulation 111, 157-164.

6. Djousse L, Arnett DK, Carr JJ, et al. (2005) Dietary linolenic acid is inversely associated with calcified atherosclerotic plaque in the coronary arteries: the National Heart, Lung, and Blood Institute Family Heart Study. Circulation 111, 2921-2926.

7. Kim HK \& Choi H (2001) Dietary alpha-linolenic acid lowers postprandial lipid levels with increase of eicosapentaenoic and docosahexaenoic acid contents in rat hepatic membrane. Lipids 36, 1331-1336.

8. Garg ML, Sebokova E, Wierzbicki A, et al. (1988) Differential effects of dietary linoleic and alpha-linolenic acid on lipid metabolism in rat tissues. Lipids 23, 847-852.

9. Ghafoorunissa, Ibrahim A \& Natarajan S (2005) Substituting dietary linoleic acid with alpha-linolenic acid improves insulin sensitivity in sucrose fed rats. Biochim Biophys Acta 1733, 67-75.

10. Weber CW, Gentry HS, Kohlhepp EA, et al. (1991) The nutritional and chemical evaluation of chia seeds. Ecol Foods Nutr 26, 119-125.

11. Bushway AA, Wilson AM, Houston L, et al. (1984) Selected properties of the lipid and protein fractions from chia seed. J Food Sci 49, 555-557.

12. Ayerza R Jr \& Coates W (2007) Effect of dietary alpha-linolenic fatty acid derived from chia when fed as ground seed, whole seed and oil on lipid content and fatty acid composition of rat plasma. Ann Nutr Metab 51, 27-34.

13. Chicco AG, D'Alessandro ME, Hein GJ, et al. (2009) Dietary chia seed (Salvia bispanica L.) rich in $\alpha$-linolenic acid improves adiposity and normalises hypertriacylglycerolaemia and insulin resistance in dyslipidaemic rats. Br J Nutr 101, 41-50.

14. Lombardo YB, Drago S, Chicco A, et al. (1996) Long-term administration of a sucrose-rich diet to normal rats: relationship between metabolic and hormonal profiles and morphological changes in the endocrine pancreas. Metabolism $\mathbf{4 5}$, 1527-1532.

15. Pighin D, Karabatas L, Rossi A, et al. (2003) Fish oil affects pancreatic fat storage, pyruvate dehydrogenase complex activity and insulin secretion in rats fed a sucrose-rich diet. J Nutr 133, 4095-4101.

16. Poudyal H, Panchal SK, Waanders J, et al. (2012) Lipid redistribution by $\alpha$-linolenic acid-rich chia seed inhibits stearoyl-CoA desaturase-1 and induces cardiac and hepatic protection in diet-induced obese rats. $J$ Nutr Biochem 23, 153-162.

17. Rossi AS, Lombardo YB, Lacorte JM, et al. (2005) Dietary fish oil positively regulates plasma leptin and adiponectin levels in sucrose-fed, insulin-resistant rats. Am J Physiol Regul Integr Comp Physiol 289, R486-R494.

18. Hein GJ, Bernasconi AM, Montanaro MA, et al. (2010) Nuclear receptors and hepatic lipidogenic enzyme response to a dyslipidemic sucrose-rich diet and its reversal by fish oil n-3 polyunsaturated fatty acids. Am J Physiol Endocrinol Metab 298, E429-E439.

19. Zimmermann R, Haemmerle G, Wagner EM, et al. (2003) Decreased fatty acid esterification compensates for the reduced lipolytic activity in hormone-sensitive lipasedeficient white adipose tissue. J Lipid Res 44, 2089-2099.

20. Ascencio C, Torres N, Isoard-Acosta F, et al. (2004) Soy protein affects serum insulin and hepatic SREBP-1 mRNA and reduces fatty liver in rats. $J$ Nutr 134, 522-529.

21. Nakatani T, Kim HJ, Kaburagi Y, et al. (2003) A low fish oil inhibits SREBP-1 proteolytic cascade, while a high-fish-oil feeding decreases SREBP-1 mRNA in mice liver: relationship to anti-obesity. J Lipid Res 44, 369-379.

22. Snedecor GW \& Cochran WG (1967) Statistical Methods, 6th ed. Ames, IA: Iowa State University Press.

23. Chicco A, D'Alessandro ME, Karabatas L, et al. (2003) Muscle lipid metabolism and insulin secretion are altered in insulinresistant rats fed a high sucrose diet. $J$ Nutr 133, 127-133.

24. Vrana A, Kazdova L, Dobesova Z, et al. (1993) Triglyceridemia, glucoregulation, and blood pressure in various rat strains. Effects of dietary carbohydrates. Ann N Y Acad Sci $\mathbf{6 8 3}, 57-68$

25. Yahagi N, Shimano H, Hasty AH, et al. (1999) A crucial role of sterol regulatory element-binding protein-1 in the regulation of lipogenic gene expression by polyunsaturated fatty acids. $J$ Biol Chem 274, 35840-35844.

26. Shimomura I, Shimano H, Korn BS, et al. (1998) Nuclear sterol regulatory element-binding proteins activate genes responsible for the entire program of unsaturated fatty acid biosynthesis in transgenic mouse liver. J Biol Chem 273, 35299-35306.

27. Nagai Y, Nishio Y, Nakamura T, et al. (2002) Amelioration of high fructose-induced metabolic derangements by activation of PPAR $\alpha$. Am J Physiol Endocrinol Metab 282, E1180-E1190.

28. Jump DB, Botolin D, Wang Y, et al. (2005) Fatty acid regulation of hepatic gene transcription. J Nutr 135, 2503-2506.

29. Pegorier JP (1998) Regulation of gene expression by fatty acids. Curr Opin Clin Nutr Metab Care 1, 329-334.

30. Kim HK (2004) Suppression of hepatic fatty acid synthase by feeding alpha-linolenic acid rich perilla oil lowers plasma triacylglycerol level in rats. J Nutr Biochem 15, 485-492.

31. Benatti P, Peluso G, Nicolai R, et al. (2004) Polyunsaturated fatty acids: biochemical, nutritional and epigenetic properties. J Am Coll Nutr 23, 281-302.

32. Jeffery NM, Newsholme EA \& Calder PC (1997) Level of polyunsaturated fatty acids and the $n-6$ to $n-3$ polyunsaturated fatty acid ratio in the rat diet alter serum lipid levels and lymphocyte functions. Prostaglandins Leukot Essent Fatty Acids 57, 149-160.

33. Neschen S, Moore I, Regittnig W, et al. (2002) Contrasting effects of fish oil and safflower oil on hepatic peroxisomal and tissue lipid content. Am J Physiol Endocrinol Metab 282, E395-E401.

34. Ide T, Kobayashi H, Ashakumary L, et al. (2000) Comparative effects of perilla and fish oils on the activity and gene expression of fatty acid oxidation enzymes in rat liver. Biochim Biophys Acta 1485, 23-35.

35. Ide $\mathrm{T}$ (2000) Effects of dietary alpha-linolenic acid on the activity and gene expression of hepatic fatty acid oxidation enzymes. Biofactors 13, 9-14.

36. Okuno M, Kajiwara K, Imai S, et al. (1997) Perilla oil prevents the excessive growth of visceral adipose tissue in rats by down-regulating adipocyte differentiation. $J$ Nutr 127, 1752-1757. 
37. Muhlhausler BS, Cook-Johnson R \& James M (2010) Opposing effects of omega- 3 and omega- 6 long chain polyunsaturated fatty acids on the expression of lipogenic genes in omental and retroperitoneal adipose depots in the rat. $J$ Nutr Metab (Epublication ahead of print version 5 August 2010).

38. Vuksan V, Jenkins AL, Dias AG, et al. (2010) Reduction in postprandial glucose excursion and prolongation of satiety: possible explanation of the long-term effects of whole grain Salba (Salvia hispanica L.). Eur J Clin Nutr 64, 436-438.
39. Vuksan V, Whitham D, Sievenpiper JL, et al. (2007) Supplementation of conventional therapy with the novel grain Salba (Salvia bispanica L.) improves major and emerging cardiovascular risk factors in type 2 diabetes: results of a randomized controlled trial. Diabetes Care 30, 2804-2810.

40. Muramatsu T, Yatsuya H, Toyoshima H, et al. (2010) Higher dietary intake of alpha-linolenic acid is associated with lower insulin resistance in middle-aged Japanese. Prev Med 50, $272-276$. 